\title{
Influence of Heterogeneous Air Entry Pressure on Large Scale Unsaturated Flow in Porous Media
}

\author{
Adam SZYMKIEWICZ ${ }^{1}$, Insa NEUWEILER ${ }^{2}$, and Rainer HELMIG ${ }^{3}$ \\ ${ }^{1}$ Gdańsk University of Technology, Department of Geotechnics, \\ Geology and Maritime Engineering, Gdańsk, Poland \\ e-mail: adams@pg.gda.pl (corresponding author) \\ ${ }^{2}$ Leibniz University Hannover,
}

Institute of Fluid Mechanics and Environmental Physics in Civil Engineering, Hannover, Germany; e-mail: neuweiler@hydromech.uni-hannover.de

${ }^{3}$ University of Stuttgart,

Institute for Modelling Hydraulic and Environmental Systems, Stuttgart, Germany, e-mail: Rainer.Helmig@iws.uni-stuttgart.de

\section{Abstract}

The paper presents numerical simulations of water infiltration in unsaturated porous media containing coarse-textured inclusions embedded in fine-textured background material. The calculations are performed using the two-phase model for water and air flow and a simplified model known as the Richards equation. It is shown that the Richards equation cannot correctly describe flow in the presence of heterogeneities. However, its performance can be improved by introducing appropriately defined effective capillary and permeability functions, representing largescale behaviour of the heterogeneous medium.

Key words: unsaturated flow, two-phase flow, heterogeneous media, air entry pressure.

\section{INTRODUCTION}

Accurate description of water flow in the unsaturated zone of soils and rocks is of paramount importance in many civil and environmental engineering 
applications. For instance, it provides us with the information necessary to investigate the safety of slopes and embankments or the fate of contaminants migrating in the subsurface. While flow in unsaturated geomaterials is essentially a two-phase process involving both water and air, it is often described using a simplified model, known as the Richards equation, which is based on the assumption of infinite mobility of the pore air. This study aims at comparison of the two-phase model and the Richards equation for vertical infiltration in soil with small scale heterogeneities.

\section{TWO-PHASE FLOW MODEL AND RICHARDS EQUATION}

In the unsaturated zone, the pore space of soils and rocks is occupied partly by water and partly by air. The flow of these fluids can be described by a two-phase model of the following form:

$$
\begin{gathered}
\frac{\partial}{\partial t}\left(\rho_{w} \theta_{w}\right)-\nabla\left(\rho_{w} \frac{k_{w}}{\mu_{w}} \nabla\left(p_{w}+\rho_{w} g z\right)\right)=0, \\
\frac{\partial}{\partial t}\left(\rho_{a} \theta_{a}\right)-\nabla\left(\rho_{a} \frac{k_{a}}{\mu_{a}} \nabla\left(p_{a}+\rho_{a} g z\right)\right)=0,
\end{gathered}
$$

where $w$ and $a$ stand for water and air, respectively, $\rho_{\alpha}$ is the density of fluid phase $\alpha, \theta_{\alpha}$ the volumetric content of the fluid phase, $\theta_{\alpha}=n S_{\alpha}, n$ the porosity, $S_{\alpha}$ the saturation, $k_{\alpha}$ the permeability for the considered fluid phase, $k_{\alpha}=k_{s} k_{r \alpha}, k_{s}$ the intrinsic permeability, $k_{r \alpha}$ the relative permeability, $\mu_{\alpha}$ the dynamic viscosity, $p_{\alpha}$ the pressure potential, $g$ the gravitational acceleration, and $z$ the elevation above the reference level. Additional relationships are needed to close the system of equations. The saturations of the two fluid phases must sum up to one:

$$
S_{w}+S_{a}=1 .
$$

The relative permeabilities are nonlinear functions of the fluid saturations. Due to the action of capillary and adsorption forces, the pressure potential of water (wetting phase) is lower than the potential of air (nonwetting phase). The difference is known as the capillary pressure or suction, $p_{c}$, and is assumed to be a function of the water saturation:

$$
p_{a}-p_{w}=p_{c}\left(S_{w}\right) .
$$

Due to the attraction of water to the solid phase, porous media remain saturated with water under suction until a specific value of the capillary pressure is exceeded. This value is known as the air entry pressure. It is inversely proportional to the diameter of the largest pores forming connected 
path through the medium. Thus, the air entry pressure of coarse-textured materials (e.g., sands), with relatively large pores, is significantly smaller than the entry pressure of fine-textured material (e.g., clays, loams).

Several analytical functions have been proposed to describe the relationships between the capillary pressure, water saturation, and the relative permeabilities of water and air in porous media. One of the often used models is the Brooks-Corey-Burdine model, which has the following form:

$$
\begin{gathered}
\Theta=\frac{\theta_{w}-\theta_{r w}}{n-\theta_{r w}-\theta_{r a}}=\left\{\begin{array}{cc}
\left(p_{c} / p_{e}\right)^{-\lambda} & p_{c}>p_{e} \\
1 & p_{c} \leq p_{e}
\end{array},\right. \\
k_{r w}=\Theta^{3+2 / \lambda}, \\
k_{r a}=(1-\Theta)^{2}\left(1-\Theta^{1+2 / \lambda}\right) .
\end{gathered}
$$

where $\Theta$ is the normalized water content or saturation, $\theta_{r w}$ and $\theta_{r a}$ the residual water and air contents, $p_{e}$ the air entry pressure, and $\lambda$ the shape parameter depending on the pore size distribution. In order to simplify the presentation but without the loss of generality we assume that the residual saturations of water and air are equal to zero.

Instead of the full two phase model, a simplified approach is often used. It is assumed that the pore air phase is infinitely more mobile than the pore water, and that it is continuous and connected to the atmosphere. Thus, the air pressure can be considered constant and equal to the atmospheric pressure. Consequently, the equation for air flow is eliminated. The capillary pressure, water saturation, and water relative permeability are uniquely defined by the water pressure. The remaining equation for water flow is known as the Richards equation. Richards equation is widely used in subsurface hydrology and geotechnics, providing water pressure and velocity fields required by contaminant transport models or soil deformation models. However, in some cases the simplifying assumptions on the behavior of the air phase are not fulfilled, which may lead to discrepancies between the results obtained with the Richards equation and the more comprehensive two-phase model. Some small differences between the models always exist, because air is not infinitely mobile. The typical water air viscosity ratio is about 50 to 60, while for a perfect agreement of the two-phase model with the Richards equation a ratio of about 100 or more is required (Tegnander 2001, Szymkiewicz 2013). Other sources of discrepancies seem to be the lack of connectivity between the pore air and the atmosphere, which may lead to air pressure buildup ahead of the infiltration front during ponded infiltration (e.g., Vachaud et al. 1973) or to formation of regions of trapped pore air (e.g., Leśniewska et al. 2009). 


\section{UPSCALED RICHARDS EQUATION FOR MEDIA WITH HETEROGENEOUS AIR ENTRY PRESSURE}

In our previous publications we showed that significant differences between the two-phase model and the Richards equation arise in a heterogeneous porous medium containing disconnected inclusions, which are characterized by a lower value of the air entry pressure than the background material. The fine-textured background becomes water-saturated for a relatively high value of suction and may block the flow of air to or from regions having lower entry pressure. In the case of infiltration, this leads to trapping of significant amount of air in coarse-textured inclusions. In the case of drainage, the coarse-textured inclusions surrounded by fine-textured matrix cannot be drained until the matrix entry pressure is exceeded. These effects were observed in laboratory experiments (Vasin et al. 2008, Szymańska 2012) and numerical simulations (Szymkiewicz et al. 2011, 2012; Szymkiewicz 2013). They can be represented using the full two-phase model, but not the Richards equation. However, this difficulty can be at least partially alleviated if the Richards equation is used with upscaled capillary and permeability functions, which take into account heterogeneity of the entry pressure.

The basic idea of the approach is shown in Fig. 1, where two sample capillary functions for the fine and coarse material were plotted together with the homogenized (upscaled) capillary functions. In the range of capillary

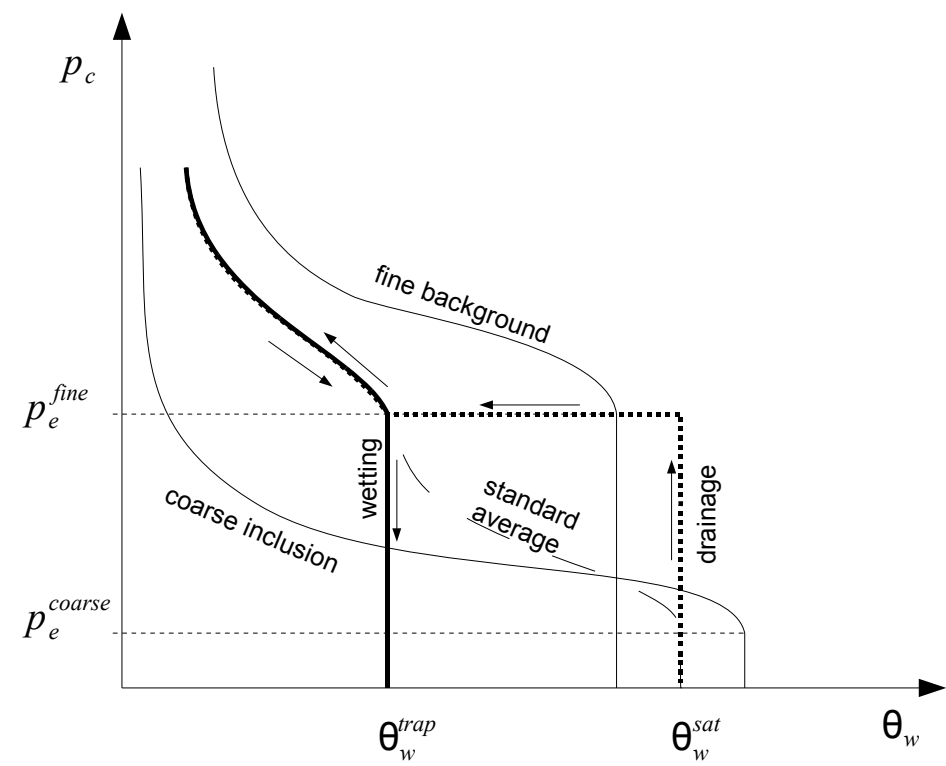

Fig. 1. Upscaled capillary functions for a heterogeneous medium with coarse inclusions. 
pressures above the entry value for the fine material (background), the same capillary pressure is assumed in the inclusions and in the background (capillary equilibrium). The upscaled water content is calculated as an arithmetic average of the water contents of the two materials, weighted by their volume fractions. However, if during wetting (infiltration) the entry pressure of the background material is reached, the inclusions remain unsaturated, while the background is fully water-saturated. Further decrease of the water suction in the background will not cause the saturation in inclusions to increase, because the air cannot leave the inclusions. On the other hand, if the medium is initially fully saturated and subjected to drainage, the air cannot invade the medium until the entry pressure for the background is exceeded. Thus, the medium remains fully saturated for the values of suction exceeding the entry pressure of inclusions. Consequently, in the range of suction values below the entry pressure of the background material the medium exhibits hysteretic behaviour, i.e., different for wetting and drainage. Note that this hysteresis is completely independent of the well-known hysteresis observed at pore scale, as it results solely from the presence of textural heterogeneities.

A similar hysteretic behaviour is observed with regard to the water permeability as a function of the capillary pressure. The effective permeability for each fluid phase is computed from the solution of the local boundary value problem representing steady state single-phase flow in the periodic cell. The permeabilities of inclusions and background are calculated as functions of the assumed capillary pressure. Since inclusions have larger intrinsic permeability than the background, the maximum possible value of the effective permeability is larger than the background permeability, but it can occur only in fully water-saturated state. The wetting process ends before full saturation is reached and the inclusions remain unsaturated and thus weakly permeable to water. As a result, one can expect the effective permeability at the end of infiltration to be smaller than the permeability of a homogeneous background material without inclusions.

Upscaled models for two phase flow with entry pressure effects were proposed by Mikelic et al. (2002), van Duijn et al. (2007), and Szymkiewicz et al. (2011). They are based on the asymptotic homogenization method and thus are applicable to media characterized by separation of micro- and macro-scale and by periodic structure. The model of Szymkiewicz et al. (2011) has been further adopted to the Richards equation (Szymkiewicz et al. 2012). The Richards equation is solved at the large scale for a homogeneous equivalent medium with effective parameters:

$$
\frac{\partial}{\partial t}\left(\rho_{w} \theta_{w}^{\mathrm{eff}}\right)-\nabla\left(\rho_{w} \frac{\mathbf{k}_{w}^{\mathrm{eff}}}{\mu_{w}} \nabla\left(p_{w}^{\mathrm{eff}}+\rho_{w} g z\right)\right)=0
$$


where the subscript "eff" denotes effective (upscaled) parameters. In the range of water pressures between zero and the negative of entry pressure for the background material, the effective water content and permeability remain essentially constant, but their values are different for wetting and drainage. In this way, the proposed formulation differs from the homogenized models for Richards equation developed earlier, e.g., by Lewandowska and Laurent (2001).

Numerical simulations for a range of problems showed that the upscaled Richards equation with effective parameters modified to account for the entry pressure effects yields better results than the fine scale solution of the Richards equation with explicit representation of the heterogeneous structure of the medium. In this paper we show further numerical investigations with focus on the problem of vertical downward infiltration and the reduction of the effective permeability of the medium due to air entrapped in inclusions.

\section{NUMERICAL SIMULATIONS}

Numerical experiments were performed for a macroscopically one-dimensional problem with two-dimensional local geometry, as shown in Fig. 2. The heterogeneous medium contains isotropic inclusions of square shape and volumetric fraction of 0.36 . Three representative sets of hydraulic parameters were chosen, which correspond to sand, loam and clay, respectively (Table 1). The following combinations were investigated: clay background with sand inclusions, clay background with loam inclusions, and loam background with sand inclusions.

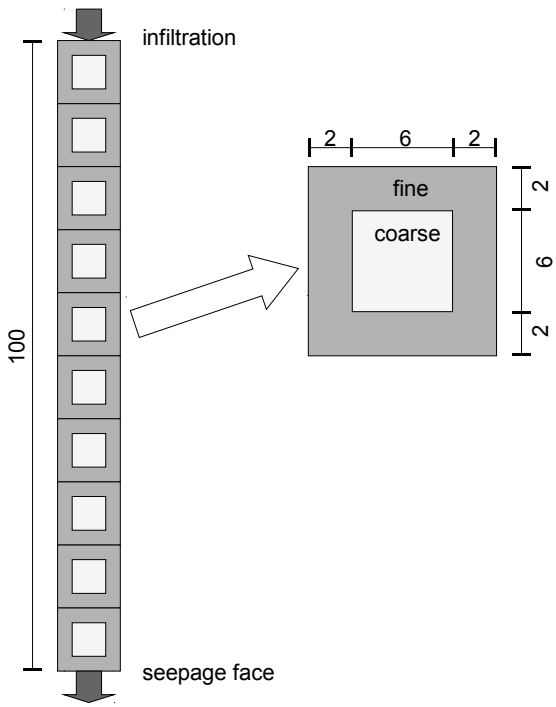

Fig. 2. Geometry of the heterogeneous porous medium. 
Table 1

Parameters of the materials used in numerical simulations

\begin{tabular}{|l|c|c|c|c|}
\cline { 2 - 5 } \multicolumn{1}{c|}{} & \begin{tabular}{c}
$n$ \\
\multicolumn{1}{c|}{}
\end{tabular} & $\begin{array}{c}p_{e} \\
{[\mathrm{~Pa}]}\end{array}$ & $\begin{array}{c}\lambda \\
{[-]}\end{array}$ & $\begin{array}{c}k_{s} \\
{\left[\mathrm{~m}^{2}\right]}\end{array}$ \\
\hline Sand & 0.4 & 1000 & 1.5 & $5 \times 10^{-10}$ \\
Loam & 0.4 & 5000 & 1.0 & $5 \times 10^{-12}$ \\
Clay & 0.4 & 10000 & 0.5 & $5 \times 10^{-14}$ \\
\hline
\end{tabular}

In each case the same initial and boundary conditions were used. Initially, the water pressure was uniform and equal to $p_{w}=-20000 \mathrm{~Pa}$ in the domain, while the air pressure was set uniformly to 0 (note that all pressure values are relative to the atmospheric pressure). It means that the medium was partially saturated and the water was under suction. At the top of the column the water pressure was instantly risen to the value $p_{w}=0$, i.e., atmospheric pressure. Consequently, the soil became fully water-saturated at the top and water was allowed to infiltrate downward. At the bottom, the seepage face boundary condition was applied. The boundary was considered impermeable to water until water pressure reached 0 . Then the condition was switched to constant pressure $p_{w}=0$, which means that the water was allowed to flow out freely from the medium. The vertical sides were impermeable to water. In the two-phase simulations, the boundary condition for air was set to atmospheric pressure at all boundaries.

For each set of materials we performed five simulations:

- background without inclusions, two-phase model (BG-2PH),

a background without inclusions, Richards model (BG-RE),

a background with inclusions, two-phase model (BG-INC-2PH),

a background with inclusions, Richards model (BG-INC-RE),

口 background with inclusions, "homogenized" Richards equation with specially assigned parameters, as described above (HOM-RE).

All numerical calculations were made using a program developed by the first author, based on a fully implicit finite volume discretization; for details see Szymkiewicz (2013). We used a uniform numerical grid of 10 cells in $x$ direction by 100 cells in $y$ direction. The time step was adjusted automatically to keep the number of Newton iterations in the range between 3 and 7 for each step.

The results can be conveniently compared in terms of the evolution of the volumetric water flux (Darcy velocity) at the outflow from the column (Figs. 3,5,7). According to the prescribed boundary condition, the flux becomes non-zero when the water pressure at the bottom of the column reaches zero. After the breakthrough, the flow tends to steady state. At the 


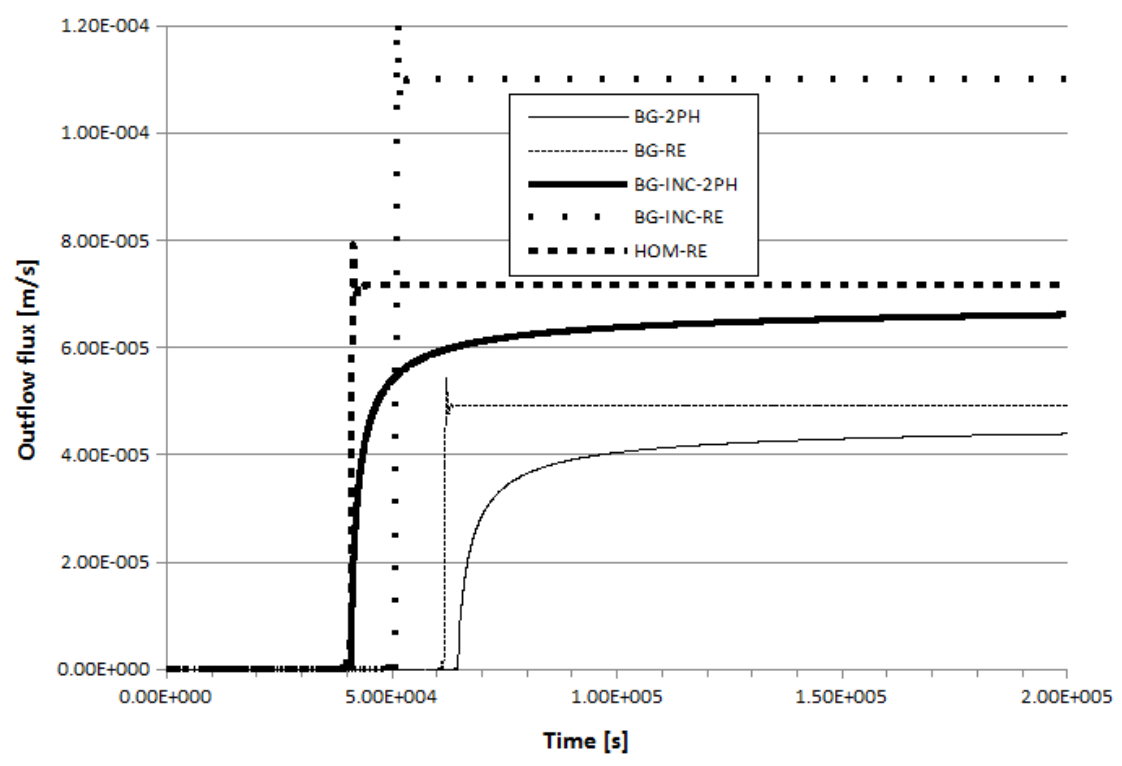

Fig. 3. Evolution of the outflow flux for clay with loam inclusions.

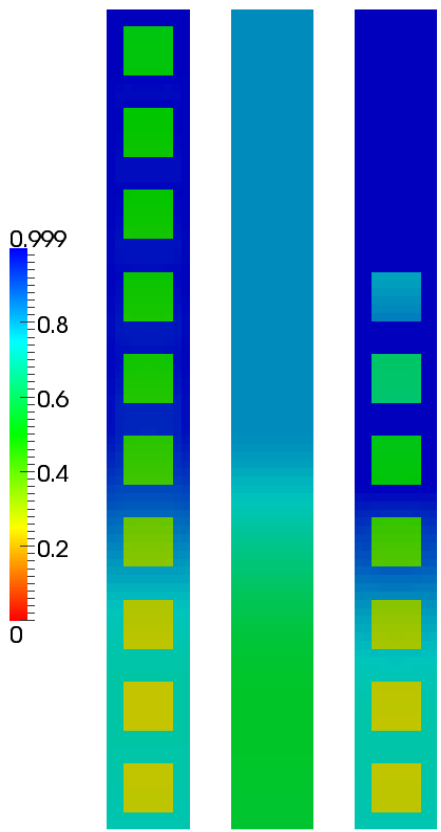

Fig. 4. Distribution of the water saturation for clay with loam inclusions after 20000 s. From left to right: BG-INC-2PH, HOM-RE, BG-INC-RE. Colour version of this figure is available in electronic edition only. 
steady state the water pressure is uniform and equal to zero in the whole column, the downward flow is only due to gravity force and the value of the flux should equal the value of the effective hydraulic conductivity at full saturation.

In Figure 3 one can see the results for clay with loam inclusions. Even for a homogeneous medium (background without inclusions) some differences between $2 \mathrm{PH}$ and RE models can be noted. The Richards equation predicts a very sharp breakthrough, with the outflow flux reaching its maximum value instantaneously, while according to the two-phase model the increase of flux is more gradual. This effect can be attributed to the presence of small amounts of air just above the outflow, which are only slowly displaced from the column. In the presence of inclusions the differences between $2 \mathrm{PH}$ and $\mathrm{RE}$ are very important. The two-phase model gives a decrease in the outflow flux with respect to the homogeneous case. This is related to the trapping of air in inclusions, which causes a decrease of the water permeability, because the inclusions are partially excluded from the water flow. Conversely, according to the solution of the Richards equation for heterogeneous medium all inclusions become fully water saturated, and consequently their presence increases the effective permeability of the medium. This behaviour is further confirmed by inspecting the distribution of the water saturation in the column according to the two phase model, the upscaled Richards equation and the fine scale Richards equation (Fig. 4).

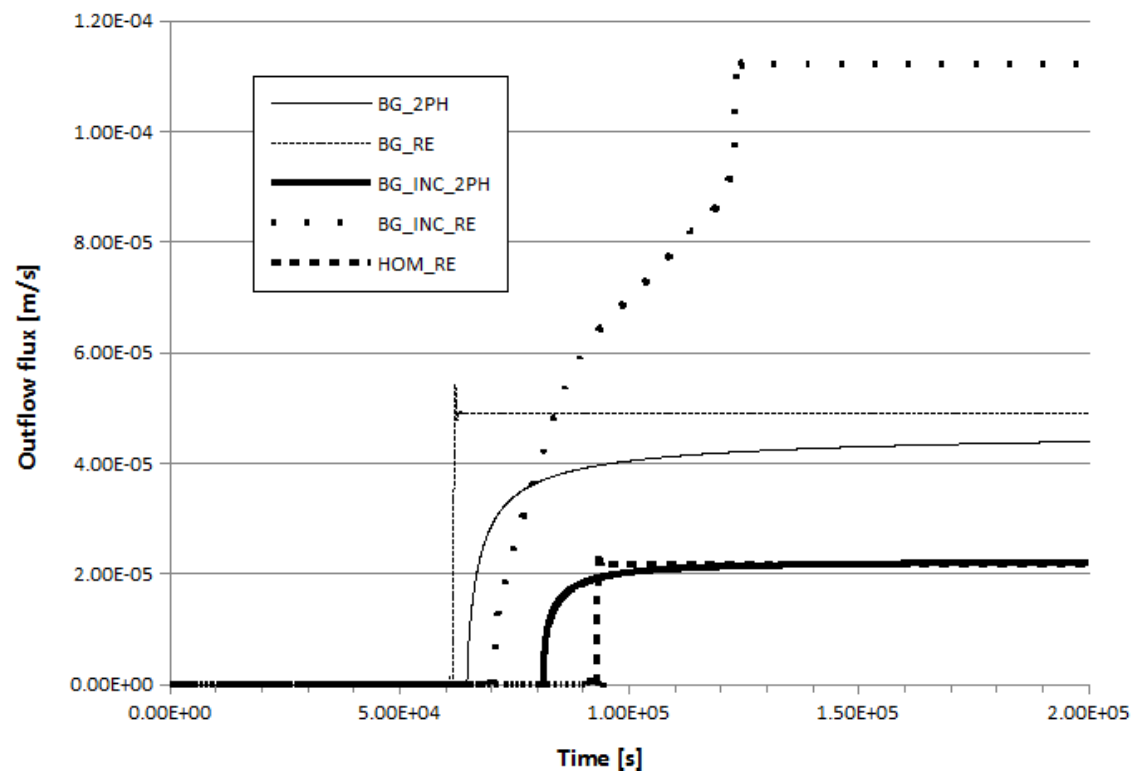

Fig. 5. Evolution of the outflow flux for clay with sand inclusions. 


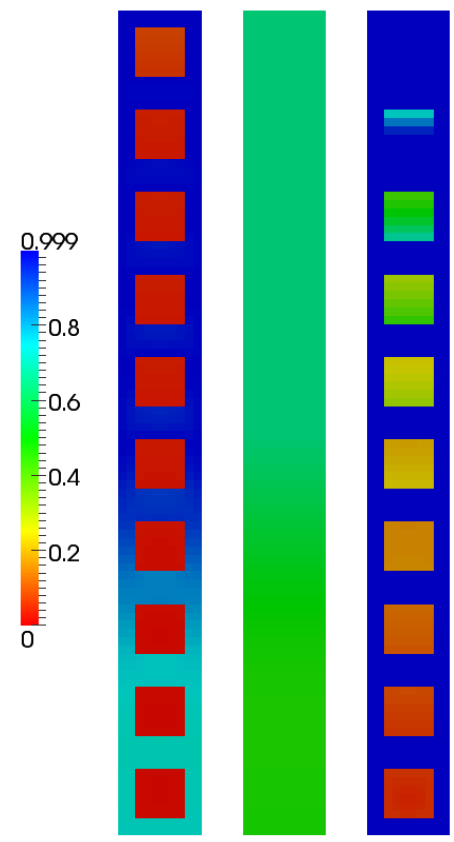

Fig. 6. Distribution of the water saturation for clay with sand inclusions after 45000 s. From left to right: BG-INC-2PH, HOM-RE, BG-INC-RE. Colour version of this figure is available in electronic edition only.

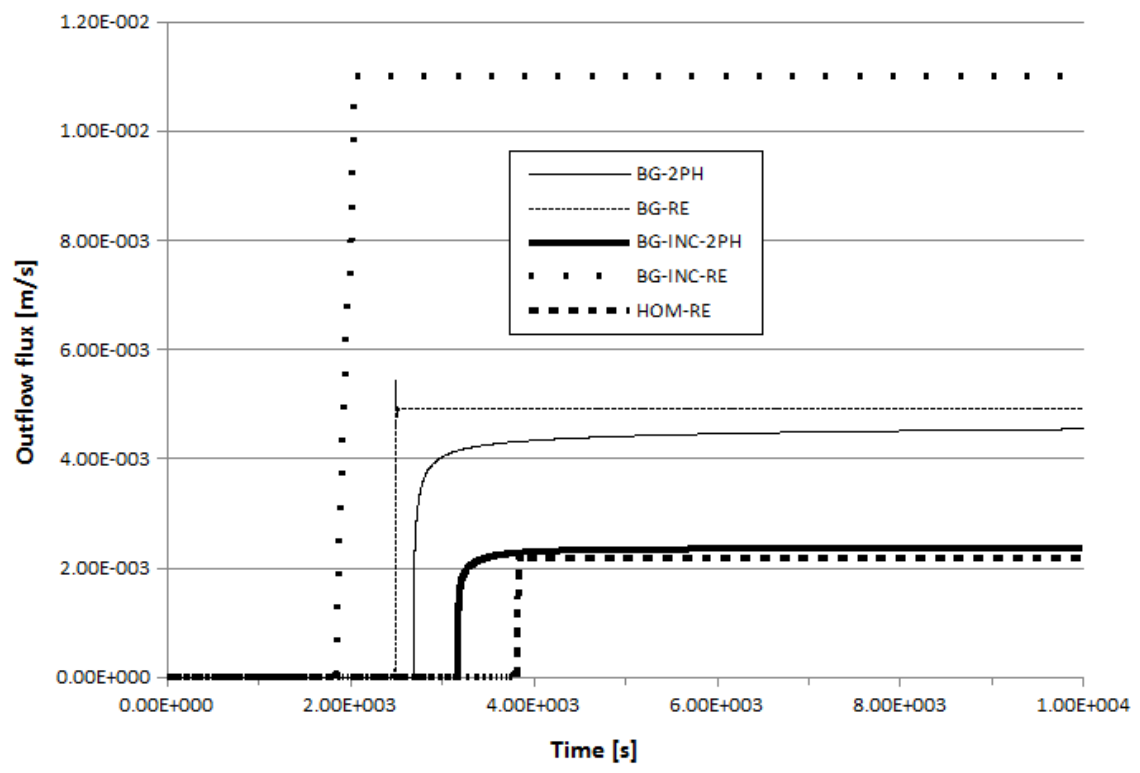

Fig. 7. Evolution of the outflow flux for loam with sand inclusions. 


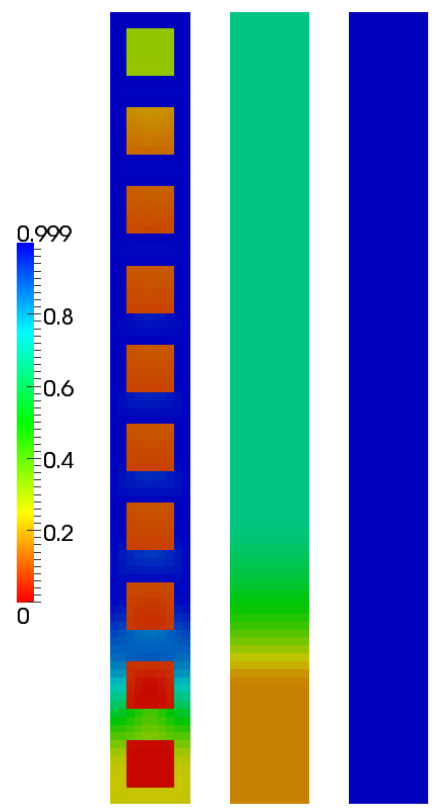

Fig. 8. Distribution of the water saturation for loam with sand inclusions after 2500 s. From left to right: BG-INC-2PH, HOM-RE, BG-INC-RE. Colour version of this figure is available in electronic edition only.

The results are shown for a transient state, before steady state is reached. It can be seen that the two-phase model results in unsaturated inclusions with trapped air, while the fine scale Richards equation predicts full saturation of inclusions. In contrast, the upscaled Richards model yields the value of the outflow flux which agrees reasonably with the result of the two-phase simulation, although the breakthrough curve is steeper. Note also that the average saturation behind the wetting front is smaller than one in the upscaled solution, which reflects air trapping in inclusions.

Similar results were obtained also for other combinations of materials, as shown in Figs. 5-8, although in these cases there are larger discrepancies between the upscaled Richards equation and the two-phase model with respect to the breakthrough time. Still, however, the upscaled model is able to represent the reduction of the effective permeability due to air trapping with reasonable accuracy.

\section{CONCLUSIONS}

Numerical simulations presented in this paper showed that the presence of local heterogeneities may lead to the trapping of pore air during downward infiltration. This effect cannot be represented by the standard Richards equa- 
tion. In particular, the Richards equation is not able to predict the reduction of the effective water permeability of the heterogeneous medium caused by the occurrence of air-filled regions. The performance of the Richards equation in the analyzed cases was significantly improved if the heterogeneous medium was replaced by an equivalent homogeneous continuum, characterized by appropriately defined capillary and permeability functions. Further investigation of the trapping phenomena is required, especially by means of laboratory experiments.

\section{References}

Leśniewska, D., H. Zaradny, P. Bogacz, and J. Kaczmarek (2009), Study of flood embankment behaviour induced by air entrapment. In: P. Samuels, S. Huntington, W. Allsop, and J. Harrop (eds.), Flood Risk Management. Research and Practice, Taylor \& Francis Group, London, 655-665.

Lewandowska, J., and J.-P. Laurent (2001), Homogenization modeling and parametric study of moisture transfer in an unsaturated heterogeneous porous medium, Transp. Porous Med. 45, 3, 321-345, DOI: 10.1023/A: 1012450327408.

Mikelic, A., C.J. van Duijn, and I.S. Pop (2002), Effective equations for two-phase flow with trapping on the micro scale, SIAM J. Appl. Math. 62, 5, 15311568, DOI: $10.1137 / \mathrm{S} 0036139901385564$.

Szymańska, P. (2012), Flow in unsaturated porous media. Numerical and experimental evaluation of the two phase model and Richards equation, M.Sc. Thesis, Gdańsk University of Technology, Faculty of Civil and Environmental Engineering, Gdańsk, Poland.

Szymkiewicz, A. (2013), Modeling Water Flow in Unsaturated Porous Media. Accounting for Nonlinear Permeability and Material Heterogeneity, Geoplanet: Earth and Planetary Sciences, Springer, Berlin Heidelberg, 237 pp., DOI: 10.1007/978-3-642-23559-7.

Szymkiewicz, A., R. Helmig, and H. Kuhnke (2011), Two-phase flow in heterogeneous porous media with non-wetting phase trapping, Transp. Porous Med. 86, 1, 27-47, DOI: 10.1007/s11242-010-9604-x.

Szymkiewicz, A., R. Helmig, and I. Neuweiler (2012), Upscaling unsaturated flow in binary porous media with air entry pressure effects, Water Resour. Res. 48, 4, W04522, DOI: 10.1029/2011WR010893.

Tegnander, C. (2001), Models for ground water flow: A numerical comparison between Richards' model and the fractional flow model, Transp. Porous Med. 43, 2, 213-224, DOI: 10.1023/A:1010749708294. 
Vachaud, G., M. Vauclin, D. Khanji, and M. Wakil (1973), Effects of air pressure on water flow in an unsaturated stratified vertical column of sand, Water Resour. Res. 9, 1, 160-173, DOI: 10.1029/WR009i001p00160.

van Duijn, C.J., H. Eichel, R. Helmig, and I.S. Pop (2007), Effective equations for two-phase flow in porous media: the effect of trapping on the microscale, Transp. Porous Med. 69, 3, 411-428, DOI: 10.1007/s11242-006-9089-9.

Vasin, M., P. Lehmann, A. Kaestner, R. Hassanein, W. Nowak, R. Helmig, and I. Neuweiler (2008), Drainage in heterogeneous sand columns with different geometric structures, Adv. Water Resour. 31, 9, 1205-1220, DOI: 10.1016/j.advwatres.2008.01.004.

Received 15 September 2013

Accepted 14 May 2014 